\section{The potential benefits of a national vaccine registry for Canada}

The article by Duong about the vaccine rollout didn't deal with a key deficiency in Canada's vaccine strategy. ${ }^{1}$ Canada continues to lack a centralized tracking system for SARS-CoV-2 vaccines. Instead, reliance is placed on a patchwork of provincial and territorial systems that are unable to communicate with Health Canada or with one another. ${ }^{2}$

Health Canada lacks vital information about population-specific vaccination rates that a national vaccine registry would provide. As a direct result, the Public Health Agency of Canada can neither compare the relative effectiveness of vaccines in different populations nor evaluate how effective each vaccine is against specific emerging variants.

A national vaccine registry would provide real-time insight into vaccine effectiveness that would prove critical to a more rapid return to normalcy. The added knowledge would enable a more agile and nuanced response to this and future outbreaks based on real-world, Canadian-specific data. This information is especially crucial when vaccine supply is limited.

Health Canada is currently siloed from the reporting on adverse events collected by the provinces and territories. ${ }^{3} \mathrm{~A}$ robust national vaccine registry would also serve as a Canada-wide repository of uncommon adverse events. The safety of vaccines within Canada's diverse populations is best understood from data mined directly from Canada's provinces and territories rather than data mainly collected outside Canada.

Finally, combining provincial and territorial data to form a robust national vaccine registry would enable the National
Advisory Committee on Immunizations to better determine the ideal interval between vaccine doses and match specific vaccines to particular variants of concern.

Yet despite the many advantages of a national vaccine registry, the implicit stance of the provinces and territories is, in fact, not to share data. ${ }^{4}$ The need for coordinated and integrated tracking systems was demonstrated by the severe acute respiratory syndrome (SARS) epidemic in 2003, after which the federal government funded Panorama to be able to manage vaccine inventory, track vaccines and adverse events, perform contact tracing and, critically, become a tool to investigate outbreaks. ${ }^{4}$ But by 2015 , the conclusion was that Panorama was not user friendly and was hard to navigate and prone to errors. ${ }^{5}$

A national vaccine registry should be created from vaccine information collected by the provinces and territories. Political will to do so at all levels of government is needed, along with commitment to ensuring the necessary resources.

Critical information is not being analyzed, even as hospital admissions and death tallies rise. The immediate creation of a national vaccine registry would save lives and prevent suffering by enabling Health Canada to provide evidence-based recommendations informed by timely, real-world, Canadian-specific data.

\section{Iris S. Gorfinkel MDCM}

General practitioner, vaccine researcher, founder and principal investigator, PrimeHealth Clinical Research, Toronto, Ont.

\section{Joel Lexchin MD}

Professor emeritus, School of Health Policy and Management, York University; emergency physician, Department of Emergency Medicine, University Health Network, Toronto, Ont.
- Cite as: CMAJ 2021 May 31;193:E808. doi: $10.1503 / \mathrm{cmaj} .78920$

\section{References}

1. Duong D. Canadian physicians frustrated with vaccine rollout. CMAJ 2021;193:E458-9.

2. Vaccination coverage in Canada. Ottawa: Government of Canada; 2019. Available: www.canada.ca/ en/public-health/services/immunization-vaccines/ vaccination-coverage.html (accessed 2021 Apr. 5)

3. Canada's Health Care System. Ottawa: Government of Canada; 2019. Available: www.canada.ca/ en/health-canada/services/health-care-system/ reports-publications/health-care-system/canada. html (accessed 2021 Apr. 5)

4. Ling J. Provinces are working with outdated vaccine tracking systems, hindering national data. Globe and Mail [Toronto]. Available: www. theglobeandmail.com/canada/article-provinces -working-with-outdated-vaccine-tracking-systems/ (accessed 2021 Apr. 5)

5. An audit of the Panorama public health system. Victoria, BC: Office of the Auditor General of British Columbia; 2015. Available: www.bcauditor .com/sites/default/files/publications/reports/ OAGBC_PanoramaReport_FINAL.pdf (accessed 2021 Apr. 5)

Competing interests: Iris Gorfinkel reports receiving research grants from GSK, Merck, Mundipharma, Johnson \& Johnson and Urovant. Dr. Gorfinkel has also received payment for lectures from GSK and CME Outfitters, and speaker fees from CBC and CTV. She has served as co-chair of the Advisory Board for Shingrix (GSK Canada). Joel Lexchin reports receiving consulting fees from Michael F. Smith, Lawyer, and Goodmans LLP. Dr. Lexchin has also received payment for being part of panels for the American Diabetes Association and Canadian Institutes of Health Research, and speaker fees from Toronto Reference Library. He is a member of the Foundation Board of Health Action International and the Board of Canadian Doctors for Medicare. He receives royalties from University of Toronto Press and James Lorimer $\&$ Co. Ltd. for books he has written.

Content licence: This is an Open Access article distributed in accordance with the terms of the Creative Commons Attribution (CC BY-NC-ND 4.0) licence, which permits use, distribution and reproduction in any medium, provided that the original publication is properly cited, the use is noncommercial (i.e., research or educational use), and no modifications or adaptations are made. See: https://creativecommons. org/licenses/by-nc-nd/4.0/ 